\title{
Communication
}

\section{Prevention and Control of the Novel Coronavirus in the Stomatological Hospital}

\author{
Peng Sun ${ }^{1, \dagger}$, Lijuan $\mathrm{Zhao}^{2, \dagger}$, Minghong Yu ${ }^{3}$, Meijuan Zhang ${ }^{4, *}$ \\ ${ }^{1}$ Department of Orthodontics, Stomatological Hospital of Yantai Affiliated to Binzhou Medical College, Yantai, China \\ ${ }^{2}$ Department of Oral and Maxillofacial Surgery, Stomatological Hospital of Yantai Affiliated to Binzhou Medical College, Yantai, China \\ ${ }^{3}$ Department of Hospital Infection-Control, Stomatological Hospital of Yantai Affiliated to Binzhou Medical College, Yantai, China \\ ${ }^{4}$ Department of Laishan, Stomatological Hospital of Yantai Affiliated to Binzhou Medical College, Yantai, China
}

\section{Email address:}

drsun1983@163.com (Peng Sun), lbz0409@sina.com (Lijuan Zhao), best32@163.com (Minghong Yu),

mjmn0108@126.com (Meijuan Zhang)

${ }^{*}$ Corresponding author

$\dagger$ Peng Sun and Lijuan Zhao co-first authors.

\section{To cite this article:}

Peng Sun, Lijuan Zhao, Minghong Yu, Meijuan Zhang. Prevention and Control of the Novel Coronavirus in the Stomatological Hospital. European Journal of Preventive Medicine. Vol. 8, No. 2, 2020, pp. 12-15. doi: 10.11648/j.ejpm.20200802.11

Received: March 7, 2020; Accepted: March 23, 2020; Published: April 21, 2020

\begin{abstract}
The 2019 novel coronavirus is a new coronavirus of the beta genus and defined as category B infectious disease, prevented as category A infectious disease. The infection of new novel coronavirus causes a diversity of clinical manifestations, including fever, dry cough, shortness of breath, muscle soreness etc. The infected are believed to be the principal source of infection, including the asymptomatic individuals who are infected. Droplet transmission and contact transmission are considered the major route of influenza spread. In some condition, aerosol transmission works as well. Alimentary tract transmission remains to be confirmed. Most of the treatment is operated in oral cavity in stomatological hospitals. The dentists, the nurses and the patients have an intimate contact with each other. By analyzing the nosocomial infection routes of novel coronavirus in stomatological hospitals, prevention and control measures were formulated to strengthen infection management, so as to control and minimize infection risks. Oral health workers should strictly implement the infection management measures in medical institutions, and do a good job in the protection of all staff, including medical care, logistics, property management and administration, the environment of the clinic, disinfection and sterilization of instruments and medical waste management, so as to prevent cross-infection and contain the spread of the epidemic.
\end{abstract}

Keywords: Stomatological Hospital, Novel Coronavirus, Infection Management

\section{Introduction}

The 2019 novel coronavirus was named when it was detected in pneumonia patients in Wuhan. National Health Commission of China defined it as category B infectious disease, prevented as category A infectious disease. The virus is a new coronavirus of the beta genus. According to New coronavirus pneumonia diagnosis and treatment plan (trial version 6), the infection causes a diversity of clinical manifestations, including fever, dry cough, shortness of breath, muscle soreness etc. Lung inflammation in imaging findings is common. Pneumonia caused by the 2019 novel coronavirus is named as "new coronavirus pneumonia", shorted as NCP in English.

The infected are believed to be the principal source of infection, including the asymptomatic individuals who are infected. Droplet transmission and contact transmission are considered the major route of influenza spread. In some condition, aerosol transmission works as well. Alimentary tract transmission remains to be confirmed. During the outpatient treatment in stomatological hospitals, we see a big population density and strong fluidity. Most of the treatment is operated in oral cavity in stomatological hospitals. The 
dentists, the nurses and the patients have an intimate contact with each other. Add all this up, we argue that it's easier for a NCP infection and carrier to infect the involved dentists and nurses than to visit a general hospital. The infected clinical workers increase the risk of the clinical visitors' infection, which makes explosive growth possible unless well controlled. To prevent cross-infection between the dentists and patients, we find out the highly possible transmission route of the new virus during oral treatment. Here are some proposals.

\section{Risk Analysis}

Droplet transmission and contact transmission are considered the major route of influenza spread. In some condition, aerosol transmission works as well. Droplet transmission works when the virus is released into the air with a sneeze, a cough even a breath of the infection. An individual at close range gets the certain amount of virus and get infected. Contact transmission works when someone gets the virus depositing at the object surface and transports the virus into oral mucosa, nasal mucosa and eye menbrane. Someone who gets infected by the virus suspended in the aerosol is defined as aerosol transmission, in this route of influenza spread confined spaces and significant time exposure help. Working in a stomatological hospital, the dentists and the nurses are surrounded with large amount of aerosol. They deserve well protection.

\subsection{Droplet Transmission}

In a stomatological hospital, the dentists get close contact with the visitors. The practice includes but not limited to clinical practice. The dentists do inquiry, clinical examination, make a treatment plan, sign informed consent and give medical advice. When they do all this above, they have face-to-face interaction. The dentists are faced with the visitor's open mouths and breathing noses during the clinical practice.

Oral treatment includes not only the teeth, but also the soft tissue such as gingival, pulp, mucosa, which are full of blood and tissue fluid. Bleeding and effusion of tissue fluid occur if the soft tissue is injured. What's worse, people salivates more than usual when treated by a dentist [1]. It makes the virus easier to spread. Dentists get in contact with the saliva, blood and tissue fluid of the patients unavoidably. Nurses get in direct contact with these when taking impression and pictures of the teeth. Both of them are in a big chance of infection. Oral instruments are in rich variety, small in size, complex in structure, which make it difficult to disinfect the oral instruments fully $[2,3]$. They recycle frequently in clinical practice. They are polluted with saliva, blood and tissue fluid. Sharp instrument injuries from the polluted instruments are seen once in a while.

\subsection{Contact Transmission}

A study has shown that the bacterial population gets significantly larger, which is attached to the mask of the dentists, the sink $1.5 \mathrm{~m}$ from the headrest of the dental chair and the computer monitor [4]. Here are common operations in dental cilinic: tooth preparation, to dry the tooth with three ways syringe, to sand blast denture bases, to do occlusal adjustment for dentures [5]. Dust mixed with virus is generated during the operations. Finally, the virus with the dust lands on the surfaces and objects nearby. It's how contact transmission works in dental cilinic [6].

\subsection{Aerosol Transmission}

Aerosol is a dispersion system of solid or liquid particles suspended in a gaseous medium. The diameter of the particles range from 0.001 to $100 \mu \mathrm{m}$. Highspeed air turbine handpiece and ultrasonic dental descaler are in common use in dental clinical practice. Taking no account of relatively low use in orthodontics, highspeed air turbine handpieces are frequently used in oral treatment. The ultrasonic dental descaler works as basics in pdriodontics. A large amount of aerosols with much virus are generated when the handpieces and descaler are at work, which contributes to the cross-infection between the workers and the visitors [7, 8]. The aerosol and splatter contamination patterns on the operators' masks, goggles, chests, gowned right arms and on the patients' goggles before and after dental treatment was invested. The result tells that contamination on every surface tested increased significantly after dental treatment, and maximum contamination was found on patients' goggles [9]. Research has shown that the air within $150 \mathrm{~cm}$ around the oral cavity is polluted with the aerosols and droplet, in particular, $50 \mathrm{~cm}$ nearby is polluted seriously [10]. In practice, we see a shorter distance than $50 \mathrm{~cm}$ between the dentists and the patients, usually a shorter distance than $150 \mathrm{~cm}$ between the dental chair and operating floor.

\section{Prevention and Control Managements for NCP in the Stomatological Hospital}

\subsection{Training on Prevention and Control Managements and Establishing the Reporting System}

All the staff are informed the managements on prevention and control, the dentists, the nurses, the cleaning personnel and the security personnel all invloed. They are taught to strengthen the awareness of prevention and control, to establish a standard process of prevention and control. Give priority to the training of the staff working in emergency department, Endodontics and Maxillofacial Surgery. The staff who have a symptom of fever, dry cough, shortness of breath or muscle soreness are encouraged to do self-report and self-isolation in the reporting system.

\subsection{Pre-check Triage Management}

The entrance and exit are guided. Everyone visiting the hospital is taken temperature by forehead thermometer, which tells the temperature without touching the body to lower the risk of cross-infection. Once there's someone who shows a rise in temperature, he is registered on the note and is guided 
to the Department fo High Fever in designated hospitals. There's at least one designated hospital in one administrative district of a city, which adopted the NCP confirmed cases and suspected cases. Tag lines on the ground in front of the registration office remind the visitors to keep others at $1 \mathrm{~m}$ 's length. The visitors enter the hall group by group. Massive crowds are avoided to keep the air flowing.

The one who takes the visitor's temperature is well protected with isolation gown, protective screen, latex gloves and surgical masks. Surgical hand antisepsis and hand-washing are necessary when he gets touch with the visitor.

\subsection{Proper Disinfection of the Clinic and Queipment}

Medical skilled and well protected dental staff accepts the urgent oral patients who need urgent treatment in the independent well-ventilated office. The dental chairs are used at intervals of one chair in the non-independent dental-chair office. Make sure all the offices are well ventilated. The offices are disinfected with ultraviolet for 1 hour twice a day. Use $1000-2000 \mathrm{mg} / \mathrm{L}$ chlorine disinfectant to wipe and disinfect the surface and ground.

After treatment, the protective eye mask and protective screen should be immersed in 1000-2000mg / L chlorine disinfectant for 30 minutes, then are rinsed with running water and dried for future use. Reusable medical devices must be cleaned, sterilized and stored in strict accordance with the requirements of WS506-2016. Single-use items are used within the validity period, and reuse is strictly prohibited. Medical waste is strictly processed in accordance with the relevant regulations.

\subsection{Patient Shunting}

Oral clinic visitors are mostly non-emergency patients with caries, malocclusion, dentition defect, cleft lip and palate or cyst. They are adviced to defer to visit the dentists. We do treatment mainly for patients with acute toothache, dental trauma, oral and maxillofacial trauma and infection.

We do consulting services for the public via internet and telephone to reduce the hospital visits. Outpatient registration is mainly by appointment to make the visit time in control, which makes queuing time for consultation significantly reduced. The registration, waiting, and payment flow are decentralized to avoid gather a crowd. Issue relevant notice to the public in advance. Do communication and interpretation for patients who have made an appointment.

\subsection{Personal Protection of Medical Staff}

The key measures for infection prevention and control come to correct selection and wearing of masks and hand hygiene. Well occupational protection and standard prevention are necessary when receiving patients for medical personnel. Medical protective masks and protective screens should be worn during operation. Use strong suction during splash operation to reduce the spread of pollutants. A 2-layer surgical surgical mask can be worn if a medical protective mask is not available. Masks must be worn in accordance with specifications. If wearing a two-layer mask, put a high level of protection inside and a low level of protection on the outer layer. Be sure not to use it overtime. Strictly follow the time indicated on the description of the mask used (general use time 4-6 hours).

Strictly implement the "two befores and three afters" principle of hand hygiene: two befores: before contacting patients, before performing aseptic operations; three afters: after contacting patients, after body fluids are exposed, and after touching the surroundings of patients. Unclean hands should not touch the mouth, nose, eyes, etc. After the operation, you should wash your hands, discard the cap and mask, and then wash your hands and take off the work clothes. Do not touch the outside of the mask when taking off the mask. Wash your hands in strict accordance with the six-step method, and wear a mask on the way home.

\section{Conclusion}

It's a critical moment of the prevention and control of NCP. Stomatologists are expected to sufficiently implement infection management measures in medical institutions. Lay emphasis on protecting all staff including medical staff, logistics, property, administration. Attach importance to clinic environment, equipment sterilization and medical waste management, etc. All these contribute to preventing cross infection and make due contributions to curb the spread of NCP.

\section{References}

[1] Li LL, Li C, Su XY, et al. (2013). Air pollution in dental clinic and intervention measures [J]. Chin J Nosocomiol, 23 (9): $2147-2148$.

[2] Fonseca G, Dourado D C, Barreto M P, et al. (2020). Antimicrobial Photodynamic Therapy (aPDT) for decontamination of high-speed handpieces: a comparative study [J]. Photodiagnosis and Photodynamic Therapy, 101686.

[3] Offner D, Brisset L, Musset A M. (2019). Evaluation of the mechanical cleaning efficacy of dental handpieces $[\mathrm{J}]$. Journal of Hospital Infection, 103 (1): e73-e80.

[4] Gu HZ, Cao WP. (2011). Levels of bacterial aerosols and their relationship with hospital infection by dental handpieces $[\mathrm{J}]$. J Oral Biomedicine, 2 (3): 145-147.

[5] Li Yajin. (2011). Cross Infection in Prothodontics Dental Clinic: A Systemic Administration Method for Precaution and Controlling [J]. Chin J Nosocomiol, 20 (3): 372-373.

[6] Li LL, Liu SJ, Wang LQ, et al. (2015). Difficulties of nosocomial infection management in dental hospital and intervention strategies $[\mathrm{J}]$. Chin $\mathrm{J}$ Nosocomiol, 25 (21): 5011-5012.

[7] Timmerman MF, Menso L, Steinfort J, et al. (2004). Atmospheric contamination during ultrasonic scaling $[\mathrm{J}]$. J Clin Periodontal, 31 (6): 458-462. 
[8] Prospero E, Savmis, Annino I. (2003). Microbial aerosol contamination of dental healthcare worker's faces and other surfaces in dental practice [J]. Infect Control Hosp Epidemiol, 24 (2): 139-141.

[9] Watanabe A, Tamaki N, Yokota K, et al. (2018). Use of ATP bioluminescence to survey the spread of aerosol and splatter during dental treatments [J]. Journal of Hospital Infection, 99 (3): 303-305.

[10] Shu XY, Lin DX, Li LL, et al. (2012). Detection and analysis of aerosols and droplets in the ultrasonic scaler [J]. Int $\mathrm{J}$ Stomatology, 39 (4): 449-45. 